\title{
La Seguridad Internacional contemporá- nea: contenidos temáticos, agenda y efectos de su ampliación ${ }^{1}$
}

\author{
Mariano Cesar Bartolomé ${ }^{2}$
}

Resumen: El campo de la Seguridad Internacional goza de indudable vigencia, debido a la importancia que adquieren las cuestiones de seguridad en los análisis sobre la situación actual y las perspectivas del tablero global. Sin embargo, no existe consenso sobre sus límites temáticos: una primera postura opina que no hubo cambios de relevancia entre la época del conflicto Este-Oeste y el presente, mientras un segundo punto de vista entiende que este campo ha incorporado más cuestiones de interés, con mayor heterogeneidad y complejidad) Este panorama nos ha llevado a establecer como objetivo principal del presente trabajo, la identificación de los rasgos preponderantes de la Seguridad Internacional contemporánea, su impacto en términos de agenda y algunos efectos de importancia que genera esta nueva situación.

Palabras clave: Amenazas, Fuerzas Armadas, Estudios de Seguridad - Seguridad Internacional

Abstract: The International Security field is still undoubtedly valid due to the importance of security issues in the analysis of today's situation and the perspectives of the global board. However, there is no consensus as to its thematic limits: there is a position which believes that there have been no changes of relevance between the Cold War and today, but a second point of view understands that this field has incorporated new issues of interest with greater heterogeneity and complexity. This state of affairs has led us to set ourselves the goal of identifying the main features of the current International Security field, its impact in terms of agenda and some important effects arising from this new situation.

Key Words: Armed Forces, International Security, Security Studies, Threats

\footnotetext{
${ }^{1}$ Recibido: 08/07/2018. Aceptado: 06/09/2018

El presente artículo fue escrito en el marco del Proyecto de Investigación J-143 "El sistema mundo en el siglo XXI y el ejercicio de la fuerza, desde los atentados del 11S hasta el conflicto de Crimea. Estudios de casos. Los medios empleados y los debates en el Derecho Internacional Público", del Programa de Incentivos de la UNLP.

${ }^{2}$ Doctor en Relaciones Internacionales. Profesor titular de la Universidad del Salvador y de la Universidad Austral. Investigador del proyecto "El sistema mundo en el siglo XXI y el ejercicio de la fuerza, desde los atentados del $11 \mathrm{~S}$ hasta el conflicto de Crimea. Estudios de casos. Los medios empleados y los debates en el Derecho Internacional Público" Proyecto J143, Instituto de Relaciones Internacionales (IRI), Universidad Nacional de La Plata (UNLP Email: marianobartolome@yahoo.com.ar
} 


\section{Introducción}

Dentro de los límites de la disciplina conocida como Relaciones Internacionales, la Seguridad Internacional configura un espacio de relevancia cuya especificidad se vincula con su particular objeto de estudio: las amenazas que penden sobre los actores del sistema internacional, y los efectos que esa situación genera. A casi tres décadas de la finalización de la Guerra Fría, este recorte disciplinar goza de indudable vigencia, debido a la importancia que adquieren las cuestiones de seguridad en los análisis sobre la situación actual y las perspectivas del tablero global. Se sostiene incluso que la seguridad se ha consolidado como una nueva guía de interpretación de la dinámica del sistema internacional, organizando y articulando sus debates en torno a fenómenos que usualmente se estudian de manera aislada (Orozco, 2006)

Aunque la vigencia e importancia de la Seguridad Internacional, en tanto recorte disciplinar, es objeto de consenso en la comunidad académica, esa coincidencia se diluye al momento de establecer sus límites en términos temáticos, existiendo dos posturas básicas en torno a este punto: mientras la primera no detecta cambios de relevancia entre la época del conflicto Este-Oeste y el presente, la restante alega que hoy la Seguridad Internacional ha experimentado importantes alteraciones en relación a tiempos pasados, tanto en términos cuantitativos (mayor cantidad de cuestiones) como cualitativos (más heterogéneas y con mayor complejidad)

Este panorama nos ha llevado a establecer, como objetivo principal del presente trabajo, la identificación de los rasgos preponderantes de la Seguridad Internacional contemporánea, y su impacto en términos de agenda, entendiendo como tal al conjunto de temas concretos que deben ser atendidos cotidianamente por las élites, en orden a la consecución de determinados objetivos en horizontes temporales concretos (Galtung, 1992) La agenda u "orden del día" nos indica quién decide y quién obedece, cómo y con respecto a qué, en el tablero global (Attiná, 2001)

A los efectos del logro de esta meta, primero se indagará sobre el concepto de Seguridad Internacional en tiempos actuales y, en caso de divergencia con los enfoques otrora vigentes, se procurará determinar la procedencia de los contenidos incorporados. En segundo término, se reparará en la traducción que tuvo la ampliación conceptual de la Seguridad Internacional en términos de agenda, con especial énfasis en tres cuestiones consideradas centrales por este autor. En tercer y último lugar, se indicarán algunos efectos de importancia que genera la nueva agenda de Seguridad Internacional, en orden a su tratamiento.

Desde una perspectiva metodológica, se realizará un enfoque lógico deductivo, con un nivel de análisis que fluctúa entre los planos descriptivo y explicativo, adquiriendo en los últimos pasajes cierto tiente normativo. La información a emplear es de tipo cualitativa, y las fuentes serán secundarias en su totalidad.

\section{La Seguridad Internacional en tiempos actuales}

Como se indicó, pese a encontrarse fuera de discusión la vigencia de la Seguridad Internacional dentro del campo de las Relaciones Internacionales, sí son objeto de debate sus alcances y contenidos, pudiéndose identificar en este punto dos posturas principales. Una primera posición, clásica, aborda el mencionado campo desde una doble pers- 
pectiva westfaliana y clausewitziana, entendiendo que su foco se encuentra en el uso de la fuerza por parte del Estado, que se convierte así en su principal objeto referente. En sus estudios sobre la cuestión, Buzan y Hansen (2009) indican que esta perspectiva se consolidó inmediatamente después de la Segunda Guerra Mundial, al punto de entenderse a la Seguridad Internacional como "el área militar especializada del realismo"

Resulta notoria la identificación de la Seguridad Internacional, en la referida perspectiva tradicional de claro sesgo estadocéntrico y clausewitziano, con el área multidisciplinar conocida como Estudios Estratégicos, que dedica su atención al empleo de la fuerza armada con fines políticos. Tomando como premisa que en un ambiente internacional anárquico los Estados deben dotarse de capacidades militares para garantizar su supervivencia y alcanzar sus intereses, los Estudios Estratégicos analizan si tales capacidades, y su uso, se adecúan a los objetivos políticos que el actor estatal pretende lograr (Jordán, 2013) También resultan nítidos sus vínculos con la Polemología, una disciplina que su mentor definió alguna vez como: "el estudio objetivo y científico de la guerra como fenómeno social susceptible de observación" (Bouthoul, 1984, p. 60) Como hemos analizado detenidamente en trabajos anteriores (Bartolomé, 2016; Bartolomé, 2017), el enfoque que propone el pensador francés opera con la matriz que se consolidó tras la Guerra de los Treinta Años, por lo cual la guerra debería entenderse en el sentido que establecen autores como Norberto Bobbio, Yoram Dinstein, Luigi Ferrajoli o incluso Martin Van Creveld, entre otros. Es decir, como un conflicto armado librado en forma abierta entre dos o más Estados a través de sus ejércitos regulares.

En tiempos recientes, la postura clásica de la Seguridad Internacional continúa concitando adherentes, quienes insisten en leerla en clave de poder militar y rechazan todo intento de redefinición, argumentando que cualquier modificación de este campo de análisis destruiría su coherencia intelectual (Del Rosso, 1995) El referente teórico más difundido tal vez sea Walt (1991), quien la asocia a "el estudio de la amenaza, uso y control de la fuerza militar". Más cerca de nuestras latitudes, Saint Pierre coincide con esos puntos de vista al indicar que la Seguridad Internacional estudia las relaciones de fuerza que se establecen entre las unidades decisorias (Estados), basadas en el mutuo reconocimiento y delimitación de las respectivas estaturas estratégicas (Saint Pierre, 2013)

Frente a la referida posición clásica, en las últimas décadas ha ganado espacio una corriente de pensamiento alternativa que rechaza la rigidez y el inmovilismo de la primera, sosteniendo que se han flexibilizado los límites y contenidos del campo de estudio en cuestión. Esta flexibilización ha sido descripta y explicada de diferentes maneras, por distintos autores, aunque en la vasta mayoría de los casos se identifica un denominador común que, en forma simplificada, consiste en la incorporación a los análisis de nuevos actores y temas alternativos, que se suman a los tradicionales (que permanecen vigentes). Dicho de otra manera, usualmente se reconocen dos ampliaciones básicas en el campo de la Seguridad Internacional contemporánea, la primera de Estados a actores no estatales, y la restante de cuestiones militares a no militares; incluso se alude a una tercera ampliación, que señala el paso del Estado al mercado como proveedor de seguridad, en clara referencia a las llamadas Compañías Militares Privadas (Krahmann, 2008)

El origen de todas estas alteraciones no ha sido otro que la reflexión académica sustentada en un renovado instrumental teórico. Las novedades registradas en el campo de las teorías de las Relaciones Internacionales a partir de principios de los años setenta, producidas a su vez por los vertiginosos cambios registrados en el escenario global, tuvie- 
ron un impacto directo en el campo de la Seguridad Internacional, hasta ese momento monopolizado por la ortodoxia realista. En este sentido, resultó clave la aparición de la idea de transnacionalidad a principios del mencionado decenio, como así también de los enfoques de Interdependencia Compleja años más tarde, en ambos casos de la mano de Keohane y Nye. Estos elementos configuran los ingredientes esenciales del planteo de Buzan (1997) de tres estadios evolutivos de la Seguridad Internacional: el primero, concordante con la visión clásica detallada en pasajes anteriores de este trabajo (vide supra), enfocado en los "estudios tradicionales de seguridad" (TSS, por sus siglas en inglés); el segundo, de ampliación de la agenda temática, consistente en la incorporación de cuestiones no planteadas en clave militar; y el último, denominado por ese autor como "estudios críticos de seguridad" (CSS), que a todo lo anterior agrega la inclusión de actores alternativos al Estado en los roles de sujeto y objeto de la seguridad, así como otras ópticas emparentadas con el Constructivismo, que se abordará más adelante (vide infra)

Otro enfoque sobre la evolución conceptual de la Seguridad Internacional coincide con Buzan en identificar tres instancias evolutivas, correspondiendo la primera a la perspectiva tradicional que prolonga su vigencia hasta inicios de los años setenta, momento en que comienza la segunda etapa signada por la ampliación de la agenda, con la incorporación a la misma de asuntos no militares; finalmente, la tercera etapa se desarrolla en el decenio siguiente y exhibe dos características centrales: por un lado, una mayor incidencia de ONGs en la conformación de la agenda temática; por otra parte, como resultado de las labores desarrolladas durante 1984 por la llamada Comisión Palme ${ }^{3}$, la irrupción del concepto Seguridad Común, enfatizando en el carácter mutuo de la seguridad y su relación con el desarrollo económico y el medio ambiente (Maciejewski, 2002)

Analizada en perspectiva histórica, la Comisión Palme tuvo un importante impacto, al postular que las principales amenazas a la Seguridad Internacional no provenían de Estados individuales, sino de problemas globales compartidos por toda la comunidad internacional. Así, puso en entredicho no sólo la concepción estadocéntrica de la seguridad, sino también la validez del criterio de no injerencia en asuntos internos (Buzan y Hansen, 2009) En cierta forma, encontramos aquí antecedentes del concepto Seguridad Humana que surgiría en el marco de la Organización de las Naciones Unidas (ONU) exactamente una década más tarde, con fuerte influencia de la perspectiva teórica constructivista, mencionada en párrafos anteriores.

El Constructivismo, al rechazar la inmutabilidad de los conceptos y sostener que éstos pueden deconstruirse y resignificarse a lo largo del tiempo, por efecto de factores culturales y sociales, ha generado importantes impactos en el campo específico de la Seguridad Internacional (Bartolomé, 2016) Precisamente, uno de ellos es la revalorización de la incidencia de los factores identitarios en determinadas cuestiones. El terrorismo proporciona un adecuado ejemplo, entre tantos posibles: abandonando discursos superficiales y lineales como el Choque de Civilizaciones que son inefectivos y producen mucha más confusión que conocimiento concreto (Halliday, 2002), el estudio de los aspectos culturales de ese flagelo desde una perspectiva sociológica y antropológica resulta clave para comprender la cosmovisión del oponente, y poder combatirlo con mayor eficacia (Badai i Dalmases, 2015)

\footnotetext{
${ }^{3}$ Se conoce así a la Comisión Independiente sobre Cuestiones de Desarme y Seguridad, dirigida por Olof Palme
} 
Por otro lado, es imposible soslayar la influencia de la cultura en la conformación de las percepciones y los conceptos de seguridad, una evidencia reconocida incluso por el pensamiento realista, resultando claves en este punto los enfoques de Katzenstein, vertidos en trabajos individuales o en obras colectivas, sobre los determinantes de la política de seguridad de un Estado (Katzenstein, 1996; Jepperson, Wendt \& Katzenstein, 1996) Una trascendencia no menor tuvo la identificación y comprensión de los procesos de "securitización", que remiten al modo en que los temas ingresan a -y egresan de- las agendas de seguridad y se interrogan sobre el rol que desempeñan en este marco los actos discursivos gubernamentales (Waever, 1999)

La cuestión de los actos discursivos estatales y sus efectos en la conformación de las realidades de seguridad, son cuestiones susceptibles de ser abordadas también desde otras perspectivas analíticas. Una de ellas es la que constituye los "estudios críticos de seguridad" (CSS) a los que alude Buzan, específicamente en su corriente danesa o Escuela de Copenhagen, donde Waever sería una figura referencial (Krause y Williams, 1997) Por otro lado, el enfoque anglosajón también conocido como Escuela Galesa, donde se destaca Booth, se aproxima al tema denunciando el carácter perimido de lo que él considera una visión ortodoxa basada en un trípode de "S" (en inglés): Estado como actor, Estabilidad como tema central (en referencia a la promoción de orden en un ambiente jerárquico) y Estrategia en alusión al empleo del instrumento militar. Frente a la obsolescencia de esa perspectiva cobra valor la idea de "emancipación", entendida como la liberación del individuo de las limitaciones físicas y sociales que le impiden hacer lo que elija hacer. Las preguntas que derivan de este planteo son cruciales: ¿Por qué existe determinada visión sobre seguridad? ¿A quién beneficia? ¿Qué ideologías o intereses sectoriales están detrás de los discursos de seguridad? (Krause y Williams, 1997)

Otra perspectiva analítica que enfocó de lleno en los actos discursivos, en materia de seguridad, es el Posestructuralismo, sea con ese nombre o en un marco más amplio de Posmodernismo. Desde un abordaje postestructuralista se subraya que las ideas (en tanto creencias, principios y actitudes que adquieren su sentido en los juegos del lenguaje en los cuales están articuladas) juegan un rol central en la construcción de "imaginarios políticos y sociales" que guían el pensar y ordenan racionalmente el mundo. Aplicado esto al tema que aquí nos ocupa: ¿Quién establece lo que es aceptable o no aceptable, justo o injusto, conveniente o inconveniente en materia de seguridad? (Moriconi Becerra, 2013) Por otro lado, los discursos intensivos en materia de seguridad, aun con las mejores intenciones, pueden generar el efecto contrario entre sus destinatarios; es decir, mayor inseguridad, expresada tanto como un miedo al futuro que bloquea las capacidades y actividades de los individuos, como un sentimiento que limita sus actitudes y aptitudes (Kernic, 2008) Frente a este cuadro de situación, se propone un "volver a pensar" carente de preconceptos, ideas apriorísticas o axiomatizadas, y hábitos experienciales (Moriconi Becerra, 2013) Dicho de otra manera, reflexionar nuevamente sobre las cuestiones de seguridad de manera creativa y libre de influencias exógenas.

Accesoriamente a lo planteado hasta aquí, los postulados constructivistas han tenido un importante efecto que no se circunscribe a las cuestiones de seguridad, siendo extensivo a todas las áreas de las Relaciones Internacionales. Esa consecuencia consiste en la erosión de cierto monopolio de las naciones centrales en lo relativo a producción teórica, facilitando la aparición de visiones alternativas, surgidas en otros contextos culturales, políticos y sociales (Tickner, 2011; Acharia, 2008; Saint Pierre, 2013) Un claro 
ejemplo en este sentido lo proporciona Ayoob (1997) al indicar que la definición tradicional de seguridad con fuerte sesgo teórico realista carece de aplicabilidad para una vasta cantidad de naciones periféricas ("subalternas", en sus términos); en esos lugares, alega el teórico, la seguridad se define en relación a las vulnerabilidades internas y externas que amenazan, o pueden amenazar, con debilitar significativamente las instituciones estatales o su presencia territorial. Esas vulnerabilidades, por su parte, se vinculan con diversos factores, entre ellos la legitimidad gubernamental a ojos de la sociedad, la cohesión social y los medios de coerción a disposición del Estado, entre otros.

La vinculación del constructivismo con la Seguridad Humana mencionada en párrafos precedentes se comprende a partir de la aparición de nuevos conceptos y abordajes que esa perspectiva teórica fomenta, o por lo menos facilita. Desde su aparición en el marco de la ONU, concretamente en el Programa de las Naciones Unidas para el DesarroIlo (PNUD), a partir del presente siglo la Seguridad Humana ha evolucionado desde su versión original con foco en el desarrollo, enfatizando en la seguridad contra amenazas crónicas y en la protección contra alteraciones súbitas del modo de vida, hacia perspectivas más vinculadas con amenazas violentas y la relativamente novedosa -y controvertidaidea de "responsabilidad de proteger", popularizada con el acrónimo R2P (Fuentes y Rojas Aravena, 2005) Un ejemplo de estas lecturas se encuentra en los análisis sobre la cantidad de decesos por desnutrición (seguridad alimentaria) y enfermedades (seguridad sanitaria) registrados en Irak luego de las sanciones económicas aplicadas por la comunidad internacional a Irak, tras la Guerra del Golfo de 1990-1991 (Achcar, 2007). Con sentido prescriptivo, se han enunciado principios de Seguridad Humana para regular los procesos de resolución de conflictos armados, desarme, desmovilización y reconstrucción de instituciones políticas (Kaldor, 2002)

La primera perspectiva de la Seguridad Humana también ha registrado avances con el paso del tiempo, entendiendo que los nuevos elementos de inseguridad que afectan a las personas requieren de un enfoque integrado. En esa línea, propone una doble estrategia de protección y potenciación de los individuos, particularmente en ciertas áreas críticas: población inocente en conflictos violentos; migrantes y desplazados; inseguridad económica; salud y educación (Fuentes y Rojas Aravena, 2005) Algunas perspectivas (Querejazu Escobari, 2011) han elevado a la Seguridad Humana a la categoría de "núcleo de la gobernanza global" que se procura, basada en el desarrollo sustentable, el respeto a los Derechos Humanos y las libertades individuales, la equidad social y la preservación ambiental. Sin embargo, como ha indicado correctamente Rivera Vélez (2008), este concepto padece una perniciosa "pretensión holística" que afecta negativamente su solidez y dificulta su puesta en práctica para la obtención de resultados concretos.

En todo caso, la Seguridad Humana no es el único concepto de relevancia que surgió al calor del impulso constructivista. Puede mencionarse también el de Seguridad Democrática, que incluso podría ser considerado una derivación del primero (específicamente de los enfoques denominados seguridad política, seguridad comunitaria y seguridad personal), si se tiene en cuenta que también fue definido por el PNUD apenas un lustro más tarde que su predecesor (Mora, 2008) Imposible omitir en este punto la idea de Seguridad Multidimensional, surgida en el marco de la declaración de Bridgetown de la Organización de Estados Americanos (OEA) del año 2002 y consagrada como enfoque común del hemisferio americano en la Conferencia Especial de Seguridad celebrada por el mencionado organismo en México un año más tarde; tanto la interpretación de sus 
contenidos -de por sí amplios- como su traducción en doctrinas en los respectivos países han sido y continúan siendo objeto de profundas controversias, existiendo versiones críticas que plantean los inconvenientes de excesivas "securitizaciones" y, sobre todo, un empleo del instrumento militar en cuestiones de índole policial (Celi, 2015)

En este escueto repaso del impacto que tuvieron en el campo de la Seguridad Internacional las novedades registradas en el campo teórico de las Relaciones Internacionales a partir de principios de los años setenta, no puede excluirse una mención al Posmodernismo. Junto con el Constructivismo, se encuentra en la perspectiva conocida como Reflectivismo, donde se ubican los abordajes teóricos no tradicionales. Aunque en ciertas circunstancias los ya mencionados enfoques postestructuralistas suelen ser asimilados a los planteos posmodernistas, estos últimos trascienden a los primeros a partir de su rasgo distintivo: la crítica al Estado, símbolo de la modernidad, como sujeto y objeto de la seguridad.

Consecuentemente, se pone en tela de juicio la suficiencia de la cartografía política tradicional de matriz westfaliana para explicar de manera efectiva el panorama de la Seguridad Internacional contemporánea; en cambio, se proponen nuevos conceptos y heterodoxas categorías de análisis geográfico, entendiendo que pueden describir y explicar de mejor manera aspectos específicos del estado de cosas actual, o al menos complementar las perspectivas tradicionales (Bartolomé, 2016) Los abordajes novedosos a los que hemos aludido, más allá de su heterogeneidad, coinciden en plantear cierta dilución de las distinciones tajantes entre interior y exterior, como lo explicita Cooper (2000), siendo que: "hay amenazas sin fronteras y fronteras sin amenazas" (Aznar, 2012, p. 165)

Es decir, no se soslaya ni subestima la geografía, que mantiene una importancia nodal: "aún en la era de los misiles intercontinentales y las bombas nucleares" (Kaplan, 2012, p. 34), aunque se evita limitar su análisis al prisma westfaliano. Trabajos recientes indican que tras el fin de la Guerra Fría un sector de la comunidad académica perdió cierto respeto por la geografía debido a la caída del Muro de Berlín, un borde artificial que indujo a creer que los condicionantes geográficos son efímeros y consecuentemente, superables; tras un breve lapso de vigencia esta concepción naïve de la política internacional, que se ha asociado con influencias teóricas liberales, ha dado paso a una etapa en la cual se le concede un nuevo respeto a los mapas (Kaplan, 2012) Además, se le ha recordado a los cándidos que la geografía es una parte fundamental tanto del por qué como del qué de las Relaciones Internacionales, sobre todo en materia de seguridad, agregando que en el siglo XXI la mayoría de los factores geográficos que ayudaron a moldear la historia continuarán incidiendo en el futuro de la política internacional (Marshall, 2017)

En esta línea de corte reivindicatorio se inscriben, por citar un caso, los alegatos en torno a la llegada de un "siglo de naciones" provocada por el fracaso de la implementación de un "siglo (norte)americano", novedad que devuelve todo su sentido a la noción de soberanía estatal (Sapir, 2008) Por otro lado, esas naciones soberanas, en función de sus intereses y capacidades -éstas últimas fluctuantes-, procurarán acceder a nuevos posicionamientos en el tablero internacional o consolidar los lugares ya obtenidos, habilitando reediciones de la tradicional "Trampa de Tucídides" en términos de potencias revisionistas y "statusquoistas" (Losurdo, 2016) Como telón de fondo, todos esos actores deberán funcionar en un contexto caracterizado por el incremento poblacional y la reducción de los recursos naturales (en especial recursos energéticos), cuyo control será 
uno de los ejes claves de la diplomacia, e incluso una de las principales causas de conflictos armados interestatales, condición similar a la registrada a fines del siglo XIX (Gray, 2004; Klare, 2001)

Vale aclarar que cualquier revisión de los criterios cartográficos vigentes impacta de manera directa en el campo de la geopolítica, siendo que -a grandes rasgos- ésta se centra en los modos en que las Relaciones Internacionales se definen en función de los factores geográficos (Marshall, 2017) Específicamente en materia de seguridad, la geopolítica se vincula de forma directa y constante con la evolución de los conflictos armados intra e interestatales, ya que a través de ellos se definen las fronteras territoriales y las soberanías nacionales (Pastor, 2005) La revisión en cuestión viene de la mano de las lecturas geopolíticas tipificadas como "críticas", que trascienden la matriz estatal y las políticas de poder distintivas de la geopolítica clásica, alcanzando de esa manera temas de análisis otrora soslayados, donde campean actores no estatales y dinámicas transnacionales; ejemplo paradigmático de lo antedicho es el abordaje geopolítico de la criminalidad organizada (Labrousse, 2011) o al terrorismo salafista (Martin, 2015; Larroque, 2016)

En esta reflexión sobre la dimensión espacial de las cuestiones de seguridad, adquiere una importancia capital el fenómeno de la globalización. La globalización incide profundamente en el campo de la Seguridad Internacional, sin que deba ser considerada una amenaza en sí misma, como ha llegado a plantearse. Como ya hemos considerado (Bartolomé, 2016), sería más correcto entenderla como un "potenciador" (IEEE, 2013) o "conductor" (Williams, 2013) de otras amenazas o riesgos, tal cual lo son el cambio climático, la urbanización descontrolada o la proliferación de ideologías radicales, entre otros. Es interesante en este sentido el enfoque de Keohane respecto a la "globalización de la violencia informal", indicando la necesaria revisión de una forma obsoleta de entender la seguridad, enmarcada únicamente en ámbitos geográficos estáticos; agrega este teórico institucionalista que la globalización de la violencia informal no es estrictamente novedosa, aunque se potenció enormemente con el abaratamiento de los costos de transporte y comunicaciones, facilitando que el territorio deje de ser concebido como un límite natural, para ser asimilado como una plataforma de proyección (Sánchez Cabarcas, 2011)

Hay otros efectos indirectos de la globalización en el campo de la seguridad, más allá de la referida potenciación de amenazas transnacionales. Eventos muy lejanos pueden incidir de manera inmediata en cualquier área del mundo, a la vez que cuestiones locales adquieren rápidamente repercusión internacional (Rojas Aravena, 2011) Además, la globalización incide en el empoderamiento de algunos actores y el debilitamiento de otros; en la influencia en rivalidades tradicionales; y en la generación de profundos cambios políticos, económicos y sociales susceptibles de derivar -a través de rechazos y resistencias- en cuadros de violencia e ingobernabilidad (Lutes, Bunn y Flanagan, 2008)

\section{Algunas cuestiones centrales de la agenda de Seguridad Interna- cional ampliada}

Hoy la Seguridad Internacional ha dejado atrás su enfoque tradicional, de aristas westfaliana y clausewitziana, ampliando sus contenidos como correlato directo de los impactos recibidos desde la Teoría de las Relaciones Internacionales. En este contexto, 
numerosas y heterogéneas cuestiones integran su agenda, por demás dinámica. A tal punto es amplia y heterogénea la agenda de la Seguridad Internacional contemporánea, que diferentes estudiosos proponen novedosos conceptos para describirla y explicarla, sirviendo como ejemplos las ideas de "inseguridad difusa" (Méndez Gutiérrez del Valle, 2011) e "incertidumbre estratégica" (Tello, 2010) En este contexto, entre los temas que se destacan por su importancia, en un breve listado que no pretende en modo alguno ser exhaustivo, consideramos la solidez institucional del Estado, las mal llamadas "nuevas amenazas", la legitimidad de las intervenciones armadas individuales o colectivas, y la fisonomía de los conflictos armados actuales. Debido a que en trabajos recientes nos hemos concentrado especialmente en esta última cuestión (Bartolomé, 2017), en este caso repararemos en las tres primeras.

Se ha alegado que la solidez de los Estados modernos está en crisis, producto de la interacción de diferentes factores, entre ellos la emergencia de nuevos actores, en un contexto de globalización; el avance de procesos de descentralización política; el fortalecimiento de entidades políticas supraestatales, finalmente, el resurgimiento de particularismos identitarios (Méndez Gutiérrez del Valle, 2011) En términos de seguridad, la referida solidez estatal está asociada a la caída sostenida de los niveles de gobernabilidad, que en determinado punto afectan negativamente el control efectivo del territorio y el monopolio de la violencia, sendos atributos básicos del Estado moderno en términos weberianos. Las primeras conceptualizaciones en torno a la remanida figura de "Estado Fallido", surgida en las postrimerías del siglo pasado, abordaron esas situaciones, aunque luego cedieron frente a otros conceptos más flexibles y menos controversiales (Zapata Callejas, 2014) Así cobra relevancia la idea de "Estados débiles", que en la lectura de Sohr (2000) pueden dividirse además en "Cuasi-Estados" y "Estados Ficticios", y que en visiones más avanzadas guardan estrecho vínculo con indicadores de naturaleza económica, política, social y de seguridad (Rice \& Patrick, 2008). La idea de "Estados Fracasados" es ambigua, fluctuando desde el derrumbe del gobierno central y la eclosión de enfrentamientos armados internos, para Hobsbawn (2006), hasta cuadros más complejos que involucran mayor cantidad de factores, para Holsti (citado en Aznar, 2012) A su turno, la pérdida de control de territorio por parte de la autoridad estatal ocupa un lugar nodal en las respectivas nociones de "Estados Frágiles" de Woodward (2006) y Kaldor (2002), aunque la teórica británica también repara en el empleo del aparato estatal por parte de las élites en beneficio propio, ignorando el interés público.

De esta manera Estados con debilidad extrema, fracasados o frágiles, aun sin pretenderlo, pueden servir como asiento de contrapoderes o "soberanías privadas" que se oponen a la soberanía estatal (Joxe, 2003), al carecer de poder efectivo para impedirlo. Esta situación se verifica actualmente con las organizaciones terroristas, agravando ese flagelo (Brito Gonçalvez \& Reis, 2017) Un efecto semejante se registra con la criminalidad organizada, cuyo surgimiento se ve favorecido por entornos sociales y espacios geográficos donde los Estados no cumplen con cierta eficacia algunas de sus funciones; en esos contextos, los criminales sustituyen al aparato estatal en el cumplimiento de esas funciones, siendo usual que esa usurpación de funciones se extienda a otras áreas (De la Corte Ibañez y Giménez Salinas, 2015) Las organizaciones criminales despliegan "mecanismos de dominación social", incluyendo la regulación de la violencia y la imposición de orden, que el Estado tácitamente puede tolerar, sea por el costo que tendría su intervención directa, por la incapacidad real para hacerlo, o incluso por el rechazo popular que podría 
generar su intromisión (Duncan, 2015) Como agravante de las situaciones descriptas, el asentamiento de organizaciones terroristas o criminales en el espacio geográfico de un Estado con debilidad extrema, fracasado o frágil promueve otro importante debate en términos de Derecho Internacional, en torno al grado de responsabilidades que le asiste al aparato estatal en cuestión, respecto a los territorios que no controla (Valasek, 2003)

En una retroalimentación negativa que conforma un círculo vicioso, la aparición y consolidación de la criminalidad conlleva erosiones adicionales a la gobernabilidad, por vía de la corrupción. En este punto, la afectación de las estructuras públicas a través de procesos de criminalización deriva en situaciones de "captura del Estado" en las cuales la organización criminal extiende su influencia corruptora sobre buena parte de la clase política, condicionando la formulación, interpretación y aplicación de las normas. En el caso específico de las drogas ilegales, el caso extremo de los procesos de criminalización exitosos es el de los llamados "narcoestados", en los cuales la economía del país depende básicamente de los ingresos procedentes de las actividades criminales en su territorio (De la Corte Ibañez y Gimenez Salinas, 2015)

Junto con el terrorismo y la criminalidad organizada, la eclosión de conflictos armados también se ve favorecida por la extrema debilidad o fragilidad del Estado, o su virtual fracaso. Lejos de explicaciones lineales, una hipótesis de trabajo en este sentido postula que en países sin estructuras estatales robustas y estables, no se observan élites políticas eficaces y transparentes que no intenten valerse de los medios a su alcance para incrementar su poder y riqueza personal, sea accediendo al aparato estatal u oponiéndose al mismo; a tal efecto, utilizan recursos ideológicos con los que explotan clivajes culturales, para conseguir seguidores y movilizar apoyos. En otros términos, una amalgama de codicia y ansias de poder, discurso ideológico y líneas divisorias étnicas y religiosas que se transforman en "líneas de ruptura" que separan amigos y enemigos, todo esto facilitado por la debilidad estatal (Múnkler, 2005)

En la etapa que Hobsbawn tipifica como el "segundo brote de barbarie" del siglo $X X$, correspondiente a los hechos de violencia y contraviolencia que signaron las últimas dos décadas de esa centuria, la desorganización civil y el derrumbe de los aparatos estatales fomentaron el incremento de la violencia, en clave cultural, particularmente étnica y confesional, derivando incluso en matanzas y genocidios sistemáticos (Hobsbawn, 2006) No es casual que un autor europeo apele al concepto de "guerras de caos" para referirse al grado de violencia que se observa en los conflictos armados resultantes de la ausencia de autoridad central, con proliferación de actores no estatales que persiguen metas criminales, políticas o religiosas, en los cuales la inseguridad lleva a la población a procurar protección al abrigo de identidades primarias, como las religiosas y étnicas (Dos Santos, 2016)

La cuestión de la legitimidad de las intervenciones armadas, unilaterales o colectivas, adquiere un lugar prioritario. Esta importancia comienza a configurarse entre fines del siglo pasado, cuando la OTAN interviene en Kosovo, y el trienio que comprende a los atentados terroristas del $11 \mathrm{~S}$ y las ulteriores operaciones militares en Afganistán e Irak. Precisamente ambos eventos, los de 1998 y 2001-2003, volvieron a poner sobre el tapete la cuestión de las llamadas "guerras justas". En esa controversia se perfilaron con cierta nitidez dos posiciones, la estadounidense que pretendía una flexibilización del Derecho Internacional y las instituciones existentes en la materia, que le garanticen un margen de acción más amplio para el empleo del poder, y la europea menos proclive al cambio, 
desde el momento en que le atribuye a ese Derecho Internacional e instituciones su éxito tras la Segunda Guerra mundial, entendiendo que un debilitamiento de ellas podría facilitar el resurgimiento de pujas de poder en sus áreas de interés (Valasek, 2003)

En una actualización de sus postulados casi tres décadas después de su primera versión (1977), Walzer ha alegado que la doctrina de la Guerra Justa no defiende ninguna guerra en concreto ni renuncia al acto bélico en sí mismo, sino que plantea que existen guerras que sí se pueden librar y hay Estados que efectivamente contemplan el empleo de la fuerza; de cara a esa situación, urge actualizar el cómo y el cuándo, para incorporar nuevos formatos y desafíos (Walzer, 2004) Los contrapuntos en torno a estas cuestiones son enormes. Walzer admite que una guerra librada contra la amenaza terrorista técnicamente podría tipificarse como justa aa; otros autores subrayan que cada vez más el terrorismo se aproxima en intensidad a una guerra, justificando y tornando legales acciones militares de Estados que en otras épocas hubieran sido inaceptables (Brito Gonçalvez \& Reis, 2017) Esta lectura registra importantes coincidencias con los planteos en torno a un "cambio de escala" del terrorismo y la guerra contemporánea, en términos de violencia, que se describirán más adelante (vide infra)

Contrario sensu, se han tomado en consideración las acciones libradas por Estados Unidos en el marco de la llamada Guerra contra el Terrorismo para rechazar empleos del poder militar basados en actitudes unilateralistas justificadas en presuntos excepcionalismos, que se racionalizan en términos de independencia y soberanía nacional (Barber, 2004) Una tercera postura, no acepta el concepto de Guerra contra el Terrorismo, independientemente de la identidad de sus protagonistas, postura que inhibe considerar a esa conducta como justa; por ejemplo, se ha tildado a esa presunta guerra como una expresión metafórica, siendo que el enemigo no está en condiciones de generar una derrota (Hobsbawn, 2006), o como la asunción schmittiana de "guerra como estado (de cosas)", una contienda permanente e indefinida en la cual el enemigo existe aunque hayan cesado las hostilidades y las operaciones militares (Pastor, 2005) Talal Asad (2008) incluso pone en tela de juicio la eventual superioridad moral de determinadas guerras frente al fenómeno terrorista, concluyendo que la idea de "guerra justa" es utilizada por Estados victoriosos para justificar su propio comportamiento; entre sus argumentos, desarrolla la idea de "escala de crueldad" según la cual la generación de muerte a civiles, la destrucción de áreas urbanas y la ruptura de la vida cotidiana generada por el Estado suele superar a la provocada por el terrorismo.

En cualquier caso, el terrorismo configura una amenaza concreta. El debate en torno a la legitimidad de las intervenciones armadas se torna aún más agudo en situaciones donde no se registra una amenaza externa tangible, sino en todo caso un cuadro de inestabilidad externa -a la fuente de intervención- que podría eventualmente afectar la estabilidad interna, globalización e interdependencia mediante. En esta línea, el empleo del instrumento militar podría evitar que problemas externos se transformen en internos, lo cual implica un desplazamiento del concepto defensa en favor del concepto protección (Joxe, 2003) Inevitablemente la cuestión deriva en los ataques armados de tipo preemptivo, o anticipatorio, que -al contrario de los ataques preventivos- no pueden ser interpretados como una necesidad imperiosa de autodefensa dirigida contra amenazas directas, inmediatas y específicas (Fontenla Ballesta, 2007)

La legitimidad de las intervenciones armadas, unilaterales o colectivas, también tiene vínculo directo con la ya mencionada y controvertida idea de "responsabilidad de 
proteger" (R2P), según la cual los Estados soberanos tienen la responsabilidad de proteger a sus ciudadanos de catástrofes -naturales o antropogénicas- evitables; en caso de no hacerlo, la comunidad internacional debe ejercer ese derecho (Fuentes y Rojas Aravena, 2005) Ese ejercicio adopta la forma de una intervención humanitaria que encuentra su primer caso paradigmático en la Operación Fuerza Aliada desarrollada por la OTAN (sin resolución de respaldo de Naciones Unidas) en Kosovo, donde el móvil fue proteger los derechos de una minoría étnica y su persecución sistemática, imponiendo el respeto a los Derechos Humanos, entendiendo que la comunidad internacional no puede permanecer impasible frente a una limpieza étnica que tiene la capacidad de detener (Sohr, 2000) De acuerdo a ciertas perspectivas críticas, la génesis de esta lectura remite a la aparición del concepto de enlargement de la mano de Anthony Lake que, en sus criterios de discriminación del espacio internacional, incluye regiones en crisis donde debe implementarse la ayuda humanitaria, como antesala de un posterior arraigo de la democracia y el sistema económico de libre mercado (Joxe, 2003) Versiones escépticas alertan que las operaciones humanitarias y la R2P podrían configurar el formato a través del cual determinados actores dotados de las capacidades necesarias, intenten expandir su modelo -o realizar una "promoción de estabilidad" en áreas donde tienen intereses, en un esquema de Seguridad Cooperativa (Cohen, 2001)-, decidiendo cuándo y cómo aplicar las resoluciones de Naciones Unidas, o incluso actuando sin ellas. "Quién nos protege de la Responsabilidad de Proteger?", plantea Losurdo (2016, p. 353) a modo de corolario.

En relación a las llamadas "nuevas amenazas" debe decirse que no existe consenso en cuanto a cuáles son exactamente los alcances del concepto. Algunas lecturas erróneas entienden que las nuevas amenazas tienen que ver con un discurso que surge en Estados Unidos para el disciplinamiento geopolítico de América Latina (Ramos, 2015) En realidad, su origen remite a la Comisión Palme y su referencia a cuestiones no necesariamente nuevas que se añaden a las amenazas tradicionales de la época de la Guerra Fría y que son vistas a través del prisma de seguridad (Kalil Mathias, 2015; Saint Pierre, 2013) En esta línea de pensamiento, en las últimas décadas ciertas amenazas han sido profundamente transformadas por diferentes factores, entre ellos la citada globalización. A modo de ejemplo, se indica que en otras épocas las amenazas a la seguridad eran estáticas, predecibles, homogéneas, con cierta rigidez y resistentes al cambio; hoy, en cambio, se caracterizan por su dinamismo, su cambio constante, su operación en red y la escasa predictibilidad que proporcionan (Schreier, 2009)

Producto de la vaguedad conceptual imperante, las jerarquizaciones en este campo abundan y presentan importantes diferencias entre sí. En lo personal, consideramos particularmente relevantes al terrorismo, el crimen organizado, la proliferación de armas de destrucción masiva (ADMs) y el conjunto de cuestiones que suelen englobarse vagamente como "ciberamenazas" o incluso "ciberguerra". En este último caso, a partir de la adaptación de los postulados de una especialista en este campo, la referencia apunta a tipos de conflictos en los que el ciberespacio es el campo de batalla, las Tecnologías de la Información y las Comunicaciones (TICS) constituyen la principal arma, las Infraestructuras Críticas $^{4}$ son particularmente vulnerables y, finalmente, cuentan con ventaja las estructuras en red (Quintana, 2016) En este sentido, no sólo se ha vuelto estratégicamente

\footnotetext{
${ }^{4}$ El concepto de Infraestructuras Críticas refiere a sistemas, máquinas, edificios e instalaciones relacionadas con la prestación de servicios esenciales (Quintana, 2016:95)
} 
relevante el ciberespacio, sino que se evidencia la perentoria necesidad de un nuevo cuerpo de teorías y doctrinas estratégicas que se refieran a la forma y el grado de respuestas "cinéticas" a los ataques virtuales (Kissinger, 2016)

En cuanto a los otros tres fenómenos mencionados, han sido caratulados como "la trinidad de amenazas preeminentes", subrayando su transnacionalidad; su capacidad para mutar en función de las circunstancias; el uso en beneficio propio de las fronteras y soberanías estatales, que constituyen barreras al accionar de los gobiernos nacionales; y su operatoria en red (Schreier, 2009) De esa trinidad, merece una especial consideración el terrorismo pues, al contrario de las otras dos amenazas preeminentes, no cuenta con una definición consensuada de la comunidad internacional, plasmada en una convención de las Naciones Unidas. Coexisten literalmente cientos de definiciones, de diferente tipo, en buena medida porque su consideración más que técnica, es moral (Silva, 2017) Con esta vaguedad conceptual, sí resulta claro que el terrorismo es más una dinámica que una estructura, y más una lógica de acción que un mero método (Brito Gonçalvez \& Reis, 2017)

Otro enfoque (Sepúlveda, 2007) coinciden en otorgarle una particular relevancia al crimen organizado y el terrorismo, destacando del primero su degradación de la capacidad estatal para garantizar la ley y el orden, facilitando la eclosión de nuevos conflictos y acciones terroristas; en el segundo caso, en tanto, enfatiza en el intenso empleo de medios de comunicación para visibilizar sus acciones ante la opinión pública, así como en el riesgo latente de empleo de ADMs. Ese eventual uso podría entenderse como el corolario de un incremento progresivo de la letalidad del fenómeno terrorista, sobre el cual -ya anticipamos en pasajes anteriores- se ha opinado que en los últimos tiempos ha experimentado un "cambio de escala" en términos de violencia que lo asemeja en cierto punto con la guerra contemporánea (Ruggiero, 2009)

La revolución registrada en las TICs se halla detrás de la operación en redes del crimen organizado y el terrorismo. En el primer caso, configuran el sustento de su constante propagación hasta alcanzar una escala virtualmente global, en lo que un autor ha dado en llamar "diáspora criminal", proceso que puede adoptar formatos de expansión, trasplante o representación (Garzón, 2013) La propagación de las actividades criminales transnacionales hasta alcanzar una escala global está acompañada por su creciente interpenetración con las actividades económicas lícitas, al punto de tornarse virtualmente imposible discriminar entre ambas esferas. Esto ha llevado a sugerir como otra especificidad de la criminalidad organizada, teniendo presente su presencia en los conflictos armados contemporáneos, su manejo tanto del capital financiero como de la violencia (Joxe, 2003) En la esfera terrorista, en tanto, ya con la irrupción de Al Qaeda comenzó a registrarse cómo este fenómeno adoptaba las estructuras celulares de los carteles de la droga y de las corporaciones de negocios virtuales, comenzando a semejar una suerte de multinacional global (Gray, 2004)

Tres apuntes se desprenden de esta novedad, siempre en referencia a la organización de Osama bin Laden: en primer lugar esa multinacional, como resultado de sus enormes capacidades, no sólo prescindió del patrocinio estatal sino que -inversamentese encontró en capacidad de patrocinar Estados, cuya conducta sutilmente logró controlar o al menos influenciar, como precisamente se constató en el caso del régimen talib de Afganistán (Ortega, 2002); segundo, se asistió de facto a la mundialización de la acción terrorista por parte de un actor que nucleaba entidades que llevaban a cabo acciones 
violentas en diferentes países, acompañando la acción central en el teatro principal (Verstrynge, 2005); en tercer lugar, los mecanismos por los cuales esas entidades autónomas se incorporaron a la red globalizada del terrorismo guardan semejanza con un sistema de "franquicias" en el cual Al Qaeda pasó a ser una marca, con un pequeño núcleo central operando como una suerte de casa matriz (Liogier, 2017)

Hoy, con la organización Estado Islámico o Daesh habiendo desplazado del centro de atención a Al Qaeda, las redes terroristas cubren el globo y están sólidamente conectadas sin ser dirigidas verticalmente, exhiben alto grado de coordinación entre sus nodos aunque éstos mantienen importantes márgenes de autonomía, y se muestran capaces de articular estrategias complejas pese a no tener estructuras centralizadas (Badai i Dalmases, 2015) El próximo paso de este proceso evolutivo consistiría, consumada la derrota de Estado Islámico y su pérdida del control territorial de su ecúmene en Medio Oriente, en su desplazamiento del plano físico a un espacio virtual desterritorializado mucho más vasto, manteniendo su influencia en la opinión pública y sus nodos mediante la difusión de su ideario a través de las TICs (Liogier, 2017)

En las diferentes manifestaciones de este novedoso terrorismo globalizado se observan dos particulares formas de asimetría, que se suman a las ya referidas precedentemente y que virtualmente anulan las ventajas militares, económicas y políticas de los actores estatales. Por un lado, una "asimetría de información", que subraya que las sociedades saben menos sobre las redes terroristas que pueden agredirlas, que las redes sobre las sociedades susceptibles de ser su blanco; por otra parte, y específicamente para el caso del terrorismo de raíz religiosa, una "asimetría de creencias" que apunta a la creciente dificultad en prevenir el accionar terrorista de organizaciones propensas al martirologio (Sánchez Cabarcas, 2011)

\section{Efectos de la ampliación de la agenda de la Seguridad Internacio- nal}

La flexibilización de los límites y contenidos del campo de estudio de la Seguridad Internacional, con la consecuente ampliación y complejización de su agenda, ha generado diferentes efectos de importancia, en lo que hace a su tratamiento. Desde el punto de vista de quien suscribe este trabajo, cuatro de esas repercusiones se destacan especialmente por su relevancia, consistiendo la primera de ellas en la revalorización de la cooperación, como herramienta clave e imposible de soslayar, a la hora de lidiar con las amenazas y riesgos que presenta el tablero global.

La cooperación es, lógicamente, el ámbito de la Seguridad Internacional donde mayor incidencia tienen los planteos teóricos liberales. Deriva de la existencia de una insoslayable "interdependencia de seguridad" (Ikenberry, 2013) y fue tildada de imprescindible por las Naciones Unidas y también por la Organización de Estados Americanos (OEA) a escala hemisférica para poder enfrentar con éxito un escenario caracterizado por amenazas diversas que no reconocen fronteras, se interconectan entre sí y se manifiestan en diferentes planos (ONU, 2004; Rojas Aravena, 2011) En este punto se ha alegado que ninguna nación más allá de su poderío ni el excepcionalismo de sus causas puede actuar unilateralmente (al menos de manera continuada) sin recurrir a la cooperación, siendo que ésta es una exigencia impuesta por la interdependencia; desde esta perspectiva, la soberanía y el poder militar deben compatibilizarse con la cooperación y el derecho (Bar- 
ber, 2004) Este imperativo de compatibilidad se torna más evidente desde el momento en que se transita una etapa internacional en la cual no se registra un país o grupo reducido de países con la capacidad de proveer el "bien público global" seguridad (Haass, 2008; Bremmer \& Roubini, 2011)

En este sentido, se ha alegado en favor de la implementación de un sistema de gobernanza global de la seguridad (Ikenberry, 2013), un escenario que guarda puntos de contacto con la imposición a escala mundial de un "paradigma de seguridad sostenible" (Abott, Rogers \& Sloboda, 2008) Pero lo cierto es que ese sistema hasta el momento no existe, ni ese paradigma se ha instalado. Frente a esa carencia, las expectativas de avance en materia de cooperación pasan en buena medida por la constitución de arquitecturas de seguridad de diferente tipo, pudiendo fluctuar desde el plano bilateral al unilateral, en un amplio rango de opciones. Mientras el formato más usual en el plano bilateral es el de las llamadas Medidas de Confianza Mutua, entendidas como acciones destinadas a prevenir situaciones de crisis y conflicto, de necesaria reciprocidad y efecto progresivo (Rojas Aravena, 2011), en el nivel multilateral las alternativas evolucionan hasta complejos modelos de Seguridad Cooperativa que incluyen acciones diplomáticas e incluso militares para promover la estabilidad en zonas externas donde pueden verse afectados los intereses de los miembros (Cohen, 2001)

La constitución de arquitecturas de seguridad es una opción que se ve facilitada por previas conformaciones de Comunidades de Seguridad. Sea en sus elaboraciones iniciales (Adler \& Barnett, 1998) o en versiones posteriores (Maciejewski, 2002), las comunidades de seguridad reconocen entre sus pilares fundamentales tanto a la existencia de opiniones y visiones compartidas que facilitan el desarrollo de confianza e identidades comunes -cuestión oportunamente subrayada por las perspectivas constructivistas-, como a la existencia de factores externos precipitantes. Estos factores, en el plano regional, constituyen el elemento central de los Complejos de Seguridad, entendiendo bajo este concepto a grupos de Estados definidos por proximidad geográfica que comparten enfoques primarios sobre seguridad (Buzan, Waever \& De Wilde, 1998)

Un segundo efecto en el tratamiento de las cuestiones de seguridad, a partir de la flexibilización de los límites y contenidos de este campo de estudio, consiste en la revalorización de nuevos enfoques, integrales, de Seguridad Nacional. Las estrategias de Seguridad Nacional refieren a las decisiones sobre el empleo de los diferentes recursos de poder del Estado para resolver sus problemas de seguridad. Hoy esas estrategias trascienden el mero empleo del instrumento militar -aunque lo incluyen- y desde esa perspectiva se entiende que se las asocie con una "gran estrategia", que será más grande cuantos más elementos incluya (Arteaga \& Fojón, 2007) Tanto en su planteo general, como en lo que hace al uso de la fuerza, la Seguridad Nacional trasciende la rígida dicotomía interior-exterior; incluye entre sus objetivos la contribución a la estabilidad global; presupone la interacción civil-militar y las respuestas integradas entre diferentes áreas de gobierno; enfatiza en la cooperación con otros países, todo esto en un marco de respeto a las formas democráticas de gobierno y los Derechos Humanos (Enseñat y Berea, 2009)

En nuestra visión, una tercera consecuencia de la ampliación y complejización de la agenda de Seguridad Internacional es la revalorización de la actividad de Inteligencia Estratégica. La fundamentación es clara, en el escenario internacional contemporáneo "el paradigma del enemigo conocido, las fronteras definidas y los objetos unívocos ha des- 
aparecido en gran manera" (Sepúlveda, 2007, p.80) Esta situación provoca incertidumbre, que se reduce a través de una adecuada labor de inteligencia correctamente interpretada por los decisores (Anguita Olmedo y Campos Zabala, 2008)

En línea con diferentes trabajos anteriores (Bartolomé, 2015; 2017), entendemos a la Inteligencia Estratégica como el producto de inteligencia para empleo del máximo poder decisorio, que trasciende a la inmediatez y la coyuntura para identificar en relación al Estado, con un horizonte temporal mayor, tanto amenazas reales o potenciales, como oportunidades. Los desafíos que presenta el actual tablero global exigen contar con avezados especialistas en cuestiones específicas, con alta capacidad interpretativa y profundos conocimientos culturales, incluso idiosincráticos, sobre la cuestión tratada. Especialistas aptos para el trabajo en equipo, para poder integrar cuerpos constituidos con finalidades analíticas concretas. Y con alto grado de adaptabilidad al cambio, siendo que en el campo de la inteligencia se pasa "de la prevención de lo conocido al management de lo desconocido" (Schreier, 2009, p.4)

Frente a un escenario caracterizado por amenazas complejas que operan transnacionalmente, muchas veces empleando en su propio beneficio las fronteras y las soberanías (en tanto obstáculos y barreras para la acción de los gobiernos), y se organizan en red, la cooperación en materia de inteligencia se ha vuelto imperativa (Anguita Olmedo y Campos Zabala, 2008; Schreier, 2009). Incluso se ha postulado como un ideal de difícil realización por el momento, la constitución de una amplia red de organismos de inteligencia para trabajar de cara a amenazas comunes y riesgos compartidos (Sepúlveda, 2007)

Finalmente, una cuarta consecuencia de la nueva agenda de Seguridad Internacional refiere a la imperativa necesidad de contar con un nuevo tipo de recursos humanos para lidiar de manera eficaz con un ambiente tan heterogéneo, complejo y dinámico. Este planteo excede a la simple profesionalización de los integrantes de las instituciones militares, pues ese concepto -surgido en los Estados modernos europeos del siglo XIXsuele circunscribirse a la apoliticidad del sector castrense, su marginación de los asuntos políticos y su distanciamiento de las instancias decisorias gubernamentales (Chaparro, 2008) Usualmente, las referencias en este sentido suelen asociarse con un nuevo perfil de soldado profesional, una cuestión que comenzó a ser objeto de estudio sobre todo a partir de las tesis de Moskos y su idea del "militar posmoderno" (Moskos, 2000) Su planteo, profundizado por otros autores, indica que en la actualidad los militares profesionales tienen que ser idóneos para interactuar con la esfera civil, desarrollar misiones no tradicionales, actuar en operaciones multilaterales e incluso integrarse en estructuras internacionales (siendo la OTAN el caso paradigmático); todo eso en un contexto de capacitación y actualización permanentes, y destrezas para el manejo de tecnologías avanzadas.

La referencia a recursos humanos aptos, empero, no se circunscribe a los militares de carrera. En ese sentido, el panorama de la Seguridad Internacional contemporánea también demanda funcionarios especializados en la conducción cívica de los asuntos de la Defensa. Funcionarios cuyo cometido esencial consista en la definición de la política y la estrategia de la Defensa y los roles que le corresponden al instrumento militar en función del contexto externo vigente; in extenso, que puedan identificar los desafíos de seguridad, cómo enfrentarlos, que capacidades se requieren y cuáles son las fortalezas y debilidades de ellas (Daly Hayes, 2008) No es casual la alusión al "control cívico" en lugar 
del trillado "control civil" originado en escritos de Huntington y Janowitz de más de medio siglo de antigüedad, pues el segundo concepto no garantiza por sí mismo que los funcionarios civiles implementen decisiones correctas. De ahí que algunos especialistas en estas cuestiones (Bruneau, Boraz \& Matei, 2008) se manifiesten en favor del concepto "Reforma del Sector Seguridad", trascendiendo a las Fuerzas Armadas y su subordinación al poder político, para indagar en torno a las amenazas existentes, las doctrinas vigentes, los roles y misiones de los organismos estatales. Por otro lado, como se desprende de su denominación, este concepto no se restringe únicamente al sector Defensa y las Fuerzas Armadas, haciéndose extensivo a todo el conjunto de instituciones de seguridad, un abordaje compatible con la idea de Seguridad Nacional anticipada previamente.

\section{Conclusiones}

A lo largo del presente trabajo, hemos intentado identificar tanto los rasgos preponderantes de la Seguridad Internacional contemporánea, como su impacto en términos de agenda. En este sentido, lo primero que se observa es que efectivamente se han modificado los límites temáticos de este recorte disciplinar en relación a los tiempos de la contienda bipolar normalmente denominada Guerra Fría. Esa modificación ha tenido un sentido expansivo y consistió en una ampliación que se expresa en la incorporación de nuevos actores y una gran cantidad de cuestiones que exhiben un alto grado de heterogeneidad entre sí. Los temas que monopolizaban este campo de estudio siguen estando presentes y gozan de vigencia, aunque carecen del estatus monopólico de otros tiempos.

Resulta claro que la ampliación temática de la Seguridad Internacional es resultado directo de la insuficiencia para explicar una realidad pródiga en amenazas que exhiben las tradicionales perspectivas westfaliana y clausewitziana, focalizadas en el uso estatal de la fuerza y entendiendo a la guerra como un conflicto armado librado en forma abierta entre dos o más Estados a través de sus ejércitos regulares. En este proceso desempeñaron un papel clave las novedades registradas en el campo de las teorías de las Relaciones Internacionales a partir de principios de los años setenta, producidas a su vez por los vertiginosos cambios registrados en el escenario global. En un listado que no pretende en modo alguno ser exhaustivo, entre esas novedades se incluyen la idea de transnacionalidad y los enfoques de Interdependencia Compleja, en ambos casos con Nye y Keohane como principales referentes; el Constructivismo, con su rechazo a la inmutabilidad de los conceptos y su particular valoración de los factores culturales y sociales; otros enfoques críticos insertos en la Escuela de Copenhage; el Posestructuralismo, con su énfasis en el rol central de las ideas en la construcción de imaginarios políticos y sociales; y el Posmodernismo y su propuesta de nuevos conceptos y heterodoxas categorías de análisis geográfico, que trasciendan la cartografía política tradicional. En relación a este último punto, el impacto se extiende incluso al ámbito de la geopolítica, que trasciende sus abordajes tradicionales para alcanzar tópicos hasta entonces desatendidos.

Bajo la influencia de la Teoría de las Relaciones Internacionales, la ampliación de contenidos de la Seguridad Internacional ha redundado en la incorporación de numerosas cuestiones a su agenda, cuya amplitud y heterogeneidad ha llevado al empleo de novedosos conceptos para describirla y explicarla. La legitimidad de las intervenciones armadas individuales o colectivas, las mal llamadas "nuevas amenazas" y la solidez institucional del Estado, se inscriben en esta perspectiva. 
Importa la solidez institucional del Estado en relación al concepto de gobernabilidad, cuya erosión acentuada deriva en cuadros de debilidad extrema, fragilidad o fracaso, favorables al asiento de organizaciones terroristas y criminales, así como al estallido de conflictos armados que suelen expresarse en términos culturales, con desmedidos niveles de violencia. Encuadrados bajo el cliché "nuevas amenazas", el terrorismo, el crimen organizado y la proliferación de armas de destrucción masiva constituyen fenómenos cuyo agravamiento no es ajeno al fenómeno de la globalización ni a la evolución de las Tecnologías de la Información y las Comunicaciones (TICs); ambos factores sustentan tanto su creciente transnacionalidad, hasta alcanzar una escala global, como su accionar en red.

El trabajo ha permitido confirmar que la prioridad de la cuestión de la legitimidad de las intervenciones armadas, unilaterales o colectivas, se vincula en primer lugar con la cuestión de las llamadas "guerras justas" y los casos que pueden ser interpretados bajo ese prisma -particularmente en relación al terrorismo-, y en segundo término con la idea de "responsabilidad de proteger" y su traducción en intervenciones humanitarias. No cabe duda que las aristas más conflictivas giran en torno al empleo discrecional de determinadas situaciones que pueden efectuar actores estatales dotados de las capacidades necesarias, para realizar intervenciones que en realidad se vinculan con intereses propios, o no consensuados por la comunidad internacional.

En suma, frente a una agenda de Seguridad Internacional que se presenta heterogénea y dinámica, a partir de la flexibilización de sus límites y contenidos, entendemos que adquieren un nuevo valor la cooperación, como herramienta clave e imposible de soslayar, a la hora de lidiar con las amenazas y riesgos que presenta el tablero global; modernos enfoques integrales de Seguridad Nacional, que van más allá de la mera cuestión de la Defensa y el empleo del instrumento militar; una Inteligencia Estratégica capaz de reducir los altos niveles de incertidumbre existentes; y recursos humanos especialmente capacitados.

La cooperación, imprescindible en un contexto donde ningún actor puede proveer el "bien público global" seguridad, parece encontrar su clave en la previa conformación de Comunidades de Seguridad. En tanto, una concepción de Seguridad Nacional ajustada a las demandas del siglo XXI proyecta sus metas más allá del ámbito local para contribuir a la estabilidad global. Una eficaz Inteligencia Estratégica demanda, como requisito previo, recursos humanos con alto grado de especialización y estructuras proclives a la cooperación. Por último, el alegato en favor de recursos humanos capacitados trasciende a los militares profesionales -a los cuales incluye- para alcanzar a funcionarios especializados en la conducción cívica de los asuntos de la seguridad, idóneos en la identificación de desafíos y amenazas, y en el diseño e instrumentación de medidas para enfrentarlas con eficiencia.

\section{Bibliografía:}

Abott C., Rogers P. \& Sloboda J. (2008). Más allá del terror. Las verdaderas amenazas que acechan al mundo. Barcelona: Los Libros del Lince

Acharia A. (2008). Nonhegemonic International Relations: A Preliminary Conceptualization. Centre for Governance and International Affairs, University of Bristol, Working Paper 10-08 
La Seguridad Internacional contemporánea - (123-145)

Achcar, G. (2007). El Choque de Barbaridades. Barcelona: Icaria

Adler E. \& Barnett M. (1998). A framework for the study of Security Communities. Cambridge International Studies Review (62), 29-66

Anguita Olmedo C. y Campos Zabala M. (2008). La globalización de la inseguridad. Revista de Relaciones Internacionales de la UNAM (101-102), 11-32

Arteaga F. y Fojón, E. (2007). El planeamiento de la política de Defensa y Seguridad en España. Madrid: Instituto Universitario Gutiérrez Mellado

Asad T. (2008). Sobre el terrorismo suicida. Barcelona: Laertes

Attiná, F. (2001). El Sistema Político Global. Buenos Aires: Paidós

Ayoob, M. (1997). Defining Security: A Subaltern Realist Perspective. En K. Krause y M. Williams (eds.), Critical Security Studies. Concepts and Cases (pp. 121-146). London: University College London Press

Aznar F. (2012). La ecuación de la guerra. Madrid: Montesinos

Badai i Dalmases, F. (2015). Orden y Desorden en el Siglo XXI. Barcelona: Icaria

Barber, B. (2004). El Imperio del Miedo. Buenos Aires: Paidós

Bartolomé M. (2016). Algunas aproximaciones a la agenda de la seguridad internacional contemporánea y la influencia teórica en sus contenidos. Política y Estrategia, (128), 101-134

Bartolomé, M. (2017). El empleo actual del concepto Guerra en las Relaciones Internacionales. Revista de Relaciones Internacionales, Estrategia y Seguridad (11)1: 43-66

Bouthoul, G. (1984). Tratado de Polemología. Sociología de las guerras. Madrid: Ediciones Ejército

Bremmer I. \& Roubini, N. (2011). A G-Zero World. Foreign Affairs (90)2

Brito Gonçalvez, J. \& Reis, M. (2017). Terrorismo. Conhecimiento e combate. Niteroi: Editora Impetus

Bruneau, T., Boraz, S. \& Matei, C. (2008).Toward a New Understanding on Civil-Military Relations. En F. Rivera Vélez (ed.), Seguridad Multidimensional en América Latina (pp. 145-151). Quito: FLACSO

Buzan, B. (1997). Rethinking Security after the Cold War. Cooperation and Conflict, 32(1), 528

Buzan B. \& Hansen L. (2009). The Evolution of International Security Studies. Cambridge: Cambridge University Press

Celi, P. (2015). La Seguridad Multifuncional en la región. En S. Alda Mejías y S. Ferreira (eds.), La Multidimensionalidad de la Seguridad Nacional: retos y desafíos de la región para su implementación (pp. 13-29). Madrid: Instituto Universitario Gutiérrez Mellado

Chaparro, J. (2008). Las Fuerzas Armadas sudamericanas y su participación en el escenario político continental. En A.Vargas Velásquez (ed.), Perspectivas actuales de la 
Seguridad y la Defensa en Colombia y en América Latina (pp.176-208). Bogotá: Universidad Nacional de Colombia

Cohen, R. (2001). Cooperative Security: from Individual Security to International Stability En

R. Cohen \& M. Mihalka (eds.), Cooperative Security New Horizons for International Order. Garmisch: The Marshall Center

Cooper, R. (2000). The Post Modern State. London: The Foreign Policy Centre

Daly Hayes, M. (2008). La profesionalización de la carrera civil de la defensa. En A. Tamayo (ed.), Conocer la guerra, construir la seguridad. Aproximaciones desde la sociedad civil (pp. 199-203). Lima: Instituto de Defensa Legal

De la Corte Ibañez, L. y Giménez Salinas, A. (2015). Crimen.Org. Barcelona: Ariel

Del Rosso, S. (1995). The Insecure State (What Future for the State?). Daedalus, 124(2):175207

Dos Santos, L. (2016). A guerra no meio de nós. A realidade dos conflitos do século XXI. Lisboa: Clube do Autor

Duncan, G. (2015). Plato o plomo. El poder político del narcotráfico en Colombia y México. México DF: Debate

Enseñat y Berea, (2009) El concepto de Seguridad Nacional en las estrategias de Seguridad Nacional. En Centro Internacional de Toledo por la Paz (ed.), Los Nuevos Paradigmas de la Seguridad (pp.9-20). Madrid: Ministerio de Defensa y CITPax

Fontenla Ballesta, S. (2007). Los campos de batalla del futuro. Madrid: Fajardo El Bravo

Fuentes, C. y Rojas Aravena, F. (2005). Promover la Seguridad Humana. Marcos éticos, normativos y educacionales en América Latina y el Caribe. París: UNESCO

Galtung, J. (1992). Geopolítica después de la Guerra Fría Un ensayo sobre la Teoría de la Agenda. En Instituto de Estudios Geopolíticos (ed.), Geoestrategia y Relaciones Internacionales (pp.49-65). Bogotá: Universidad Militar Nueva Granada

Garzón, J. (2013) La Diáspora Criminal La difusión transnacional del crimen organizado y cómo contener su expansión (pp.1-26). En J. Garzón y E. Olson (eds.), La Diáspora Criminal. Washington DC: Woodrow Wilson Center

Gray, J. (2004). Al Qaeda y lo que significa ser moderno. Buenos Aires Paidós

Haass, R. (2008). La era de la no polaridad. Foreign Affairs Latinoamérica, 8(3): 66-78

Halliday, F. (10 de marzo 2002). New World, but the same Old Disorder. The Observer.

Hobsbawn, E. (2006). Guerra y Paz en el Siglo XXI. Barcelona: Crítica

Instituto Español de Estudios Estratégicos [IEEE] (2013). Los potenciadores de riesgo. Madrid: Ministerio de Defensa

Ikenberry, J. (2013) La cooperación para la seguridad mundial en el siglo XXI. En J. Stiglitz y M. Kaldor (eds.). La búsqueda de la seguridad. Protección sin proteccionismo y el desafío de la gobernanza global (pp.127-154). Buenos Aires: Paidós

Jepperson, R., Wendt, A. \& Katzenstein, P. (1996). Norms, Identity and Culture in National 
Security. En P. Katzenstein (ed.) The Culture of National Security: Norms and Identity in World Politics (pp. 33-77). New York: Columbia University Press

Jordán, J. (2013). Manual de Estudios Estratégicos y Seguridad Internacional. Madrid: Plaza y Janés

Joxe, A. (2003). El Imperio del Caos. Buenos Aires: Fondo de Cultura Económica

Kaldor, M. (2002). Haz la ley y no la guerra. La aparición de la Sociedad Civil Global. En M. Castells y N. Serra (Eds.), Guerra y Paz en el Siglo XXI (pp. 67-98). Barcelona: Kriterios-Tusquets

Kaplan, R. (2012). The Revenge of Geography. New York: Random House

Katzenstein, P. (1996). Introduction: Alternatives Perspectives on National Security. En P. Katzenstein (ed.) The Culture of National Security: Norms and Identity in World Politics (pp. 33-77). New York: Columbia University Press

Kernic, F. (2008). La producción de inseguridad en la sociedad global En C. Navajas Zubeldía y D. Iturriaga Barco (eds.), Crisis, dictaduras, democracia (pp 71-78). Logroño: Universidad de La Rioja

Khalil Mathias, S. (2015). El desarrollo como variable de Seguridad Multidimensional en perspectiva regional: el caso de América del Sur. En S. Alda Mejías y S. Ferreira (eds.), La Multidimensionalidad de la Seguridad Nacional: retos y desafíos de la región para su implementación (pp. 53-53). Madrid: Instituto Universitario Gutiérrez Mellado

Kissinger, H. (2016). Orden Mundial. Barcelona: Debate

Klare, M. (2001). Resource Wars: The New Landscape of Global Conflict. Nueva York. Henry Holt/Metropolitan

Krahmann, E. (2008). Security. Collective Good or Commodity? European Journal of International Relations, 14(3), 379-404

Krause, K. \& Williams, M. (1997). From Strategy to Security: Foundations of Critical Security Studies. En K. Krause y M. Williams (eds.), Critical Security Studies. Concepts and Cases (pp.33-59). London: University College London Press

Labrousse, A. (2011). Geopolítica de las Drogas. Buenos Aires: Editorial Marea

Larroque, A. (2016). Geopolítica de los Islamismos. Madrid: RIALP

Liogier, R. (2017). La guerra de civilizaciones no tendrá lugar: coexistencia y violencia en el Siglo XXI. Salamanca: Comunicación Social

Losurdo, D. (2016). Un mundo sin guerras. La idea de paz, de las promesas del pasado a las tragedias del presente. Barcelona: El Viejo Topo

Lutes, C., Bunn, E. \& Flanagan, S. (2008). The Emerging Global Security Environment. En S. Flanagan y J. Schear (eds.) Strategic Challenges (pp.1-19). Washington DC: National Defense University Press \& Potomac Books

Maciejewski W. (2002). The Baltic Sea Region. Cultures, Politics, Societies. Uppsala: Baltic University Press 
Marshall, T (2017). Prisioneros de la Geografía. Barcelona: Ediciones Península

Martín J. (2015). Estado Islámico. Geopolítica del caos. Madrid: Libros de la Catarata

Méndez Gutiérrez del Valle, R. (2011). El Nuevo Mapa Geopolítico del Mundo. Valencia: Tirant Lo Blanch

Mora, A. (2008). Seguridad y democracia ¿concepciones antagónicas? En A.Vargas Velásquez (ed.), Perspectivas actuales de la Seguridad y la Defensa en Colombia y en América Latina (pp.13-21). Bogotá: Universidad Nacional de Colombia

Moriconi Becerra, M. (2013). Ser violento. Los orígenes de la inseguridad y la víctimacómplice. Buenos Aires: Capital Intelectual

Moskos, C., Williams J. \& Segal D. (2000). The Postmodern Military. Armed Forces after the Cold War. New York \& Oxford: Oxford University Press

Münkler H. (2005). Viejas y nuevas guerras. Asimetría y privatización de la violencia. Madrid: Siglo XXI

Organización de las Naciones Unidas (2004). Un mundo más seguro: la responsabilidad que compartimos. Informe del Grupo de Alto Nivel sobre las amenazas, los desafíos y el cambio, A/59/565. Nueva York: Organización de las Naciones Unidas

Orozco, G. (2006). El concepto de Seguridad en la Teoría de las Relaciones Internacionales. Revista CIDOB d'Afers Internacionals (72), 161-180

Pastor, J. (2005). Geopolítica, guerra y Balcanes globales. En J. Brandáriz (ed.), Guerra global permanente. La nueva cultura de la inseguridad (pp. 15-50). Madrid: Editorial de la Catarata

Ortega A. (2002). Golpes a la globalización. En M. Castells y N. Serra (Eds.), Guerra y Paz en el Siglo XXI (pp. 149-153). Barcelona: Kriterios-Tusquets

Querejazu Escobari, A. (2011) Gobernanza global y Estado, desterritorialización y desvanecimiento de la autoridad. En H. Godoy, H. González Arana y G. Orozco Restrepo (eds.), Construyendo lo global. Aportes al debate de Relaciones Internacionales (pp. 35-61) Barranquilla: Editorial Universidad del Norte

Quintana, Y. (2016). Ciberguerra. Madrid: Editorial de la Catarata

Rice S. \& Patrick S. (2008). State Weakness in the Developing World. Washington DC: The Brookings Institution

Rivera Vélez, F. (2008). Estudio introductorio. Seguridad Multidimensional en América Latina. En F. Rivera Vélez (ed.), Seguridad Multidimensional en América Latina (pp. 11-35). Quito: FLACSO

Rojas Aravena, F. (2011). Seguridad Internacional, el espacio y posición de América Latina. En Instituto Español de Estudios Estratégicos (ed.), Los desafíos de la Seguridad en Iberoamérica (pp.19-82). Madrid: Ministerio de Defensa

Ruggiero, V. (2009). La Violencia Política. Barcelona: Antrophos Editorial

Saint Pierre, H. (2013). "Defesa" ou "Segurança"? Reflexões em torno de conceitos e ideologías. En E. Mei y H. Saint Pierre (Eds.), Paz e Guerra. Defesa e segurança en- 
tre as nações (pp. 11-38). São Paulo: Editora UNESP

Sánchez Cabarcas, F. (2011). Seguridad en Medio Oriente: efectos del 11S y Teorías de Relaciones Internacionales. En H. Godoy, H. González Arana y G. Orozco Restrepo (eds.), Construyendo lo global. Aportes al debate de Relaciones Internacionales (pp. 299-321) Barranquilla: Editorial Universidad del Norte

Sapir, J. (2008). El nuevo Siglo XXI. Del siglo americano al retorno de las naciones. Barcelona: EI Viejo Topo

Schreier F. (2009). Fighting the Pre-eminent Threats with Intelligence-led Operations. Geneva Centre for the Democratic Control of Armed Forces (DCAF), Occasional Paper No 16

Sepúlveda I. (2007). La Seguridad Internacional ante las Nuevas Amenazas. En Programa de las Naciones Unidas para el Desarrollo (ed.), Defensa Nacional: dimensiones internacionales y regionales (pp.63-81). Buenos Aires: PNUD

Silva, L. (2017). Vidas Armadas. Santiago de Chile: Planeta

Sohr, R. (2000). Las guerras que nos esperan. Santiago de Chile: Ediciones B

Tello, A. (2010). La Incertidumbre Estratégica. En M. Bartolomé (ed.), Seguridad y Defensa en tiempos de Bicentenario. Visiones desde Argentina y Chile (pp.21-34).

Buenos Aires: Instituto de Publicaciones Navales

Tickner, A. (2011). Relaciones de conocimiento Centro-Periferia: hegemonía, contribuciones locales e hibridización. En H. Godoy, H. González Arana y G. Orozco Restrepo (eds.), Construyendo lo global. Aportes al debate de Relaciones Internacionales (pp. 18-34) Barranquilla: Editorial Universidad del Norte

Valasek, T. (2003). New Threats, New Rules. Revising the Law of War. World Policy Journal, Spring, 17-24

Verstrynge, J. (2005). La guerra periférica y el Islam revolucionario. Barcelona: El Viejo Topo

Waever, O. (1999) Securitization and Desecuritization. En R. Lipschutz (ed.), On Security (pp.46-86). New York: Columbia University Press

Walt, S. (1991). The Renaissance of Security Studies. International Studies Quarterly, 35(2), 211-239

Walzer, M. (2004). Reflexiones sobre la Guerra. Barcelona: Paidós

Williams, P. (2013). Lawlessness and Disorder: An Emerging Paradigm for the $21^{\text {st }}$ Century. En M. Miklaucic, J. Brewer \& G. Barnabo (eds.), Convergence. Illicit Networks and National Security in Age of Globalization (pp.15-36). Washington DC: National Defense University Press

Woodward, S. (2006) Fragile States: Exploring the Concept. Madrid: FRIDE

Zapata Callejas J. (2014). La teoría del “Estado fallido”: entre aproximaciones y disensos. Revista de Relaciones Internacionales, Estrategia y Seguridad (9)1, 87-110 